\title{
A New Approach and Analysis of Modeling the Human Body in RFID-Enabled Body-Centric Wireless Systems
}

\author{
Karoliina Koski, ${ }^{1}$ Toni Björninen, ${ }^{1}$ Lauri Sydänheimo, ${ }^{1}$ \\ Leena Ukkonen, ${ }^{1}$ and Yahya Rahmat-Samii ${ }^{2}$ \\ ${ }^{1}$ Wireless Identification and Sensing Systems Research Group, Department of Electronics and Communications Engineering, \\ Tampere University of Technology, 33720 Tampere, Finland \\ ${ }^{2}$ Department of Electrical Engineering, University of California, Los Angeles, CA 90095, USA
}

Correspondence should be addressed to Karoliina Koski; karoliina.koski@tut.fi

Received 2 October 2013; Revised 10 March 2014; Accepted 12 March 2014; Published 24 April 2014

Academic Editor: Young Joong Yoon

Copyright (C) 2014 Karoliina Koski et al. This is an open access article distributed under the Creative Commons Attribution License, which permits unrestricted use, distribution, and reproduction in any medium, provided the original work is properly cited.

Body-centric wireless systems demand wearable sensor and tag antennas that have robust impedance matching and provide enough gain for a reliable wireless communication link. In this paper, we discuss a novel and practical technique for the modeling of the human body in UHF RFID body-centric wireless systems. What makes this technique different is that we base the human model on measured far-field response from a reference tag attached to the human body. Hereby, the human body model accounts for the encountered human body effects on the tag performance. The on-body measurements are fast, which allows establishing a catalog of human body models for different tag locations and human subjects. Such catalog would provide a ready simulation model for a wide range of wireless body-centric applications in order to initiate a functional design. Our results demonstrate that the suggested modeling technique can be used in the design and optimization of wearable antennas for different real-case body-centric scenarios.

\section{Introduction}

Wireless monitoring of humans by means of low-power and low-cost UHF (860-960 MHz) radio frequency identification (RFID) is rapidly emerging. An RFID body-centric wireless system will provide several new and important features for applications in wireless sensor systems, healthcare, realtime remote biomonitoring of human vital signs, and neural activity [1-9]. One of the open challenges is to create efficient wearable antennas for RFID tags and sensors in close vicinity of the human body. The wearable antenna is a key component of the body-centric system as it provides the wireless communication link between body-worn electronics and base stations located in the surrounding. For the widespread use of wearable antennas to become reality, the wearable antenna should be unobtrusively comfortable to wear, inexpensive, and totally maintenance-free [10].

Previously, the human body effects on antenna performance have been assessed by means of measurements and computational tools $[5,11,12]$. The human body models based on dielectric material parameters measured from tissue samples [13] have been developed with varying level of detail, from multilayered $[5,11]$ to homogenous $[8,9]$ structures. In [14], an on-skin passive UHF RFID tag is designed and tuned directly onto skin with no intervening substrate using a 4layered model of human tissue. Electrical data for the tissue layers are taken from tissue database.

Our novel method for modeling the human body is intended to overcome the need for knowing the electrical properties of specific biological tissues in detail. Additionally, the goal is to provide a method that can be easily and quickly adopted in practice for different body-centric scenarios.

We first consider the measured response from a reference RFID tag attached to a human volunteer subject as the true tag response. This includes not only the effect of high permittivity and dissipative tissues but also effects of polarization between tag and reader antennas, air between the tag and the body, uneven body surface, and shadowing. Such effects are easily overlooked, or even omitted, when too small and simplified phantoms are used for the measurement purpose. We then 


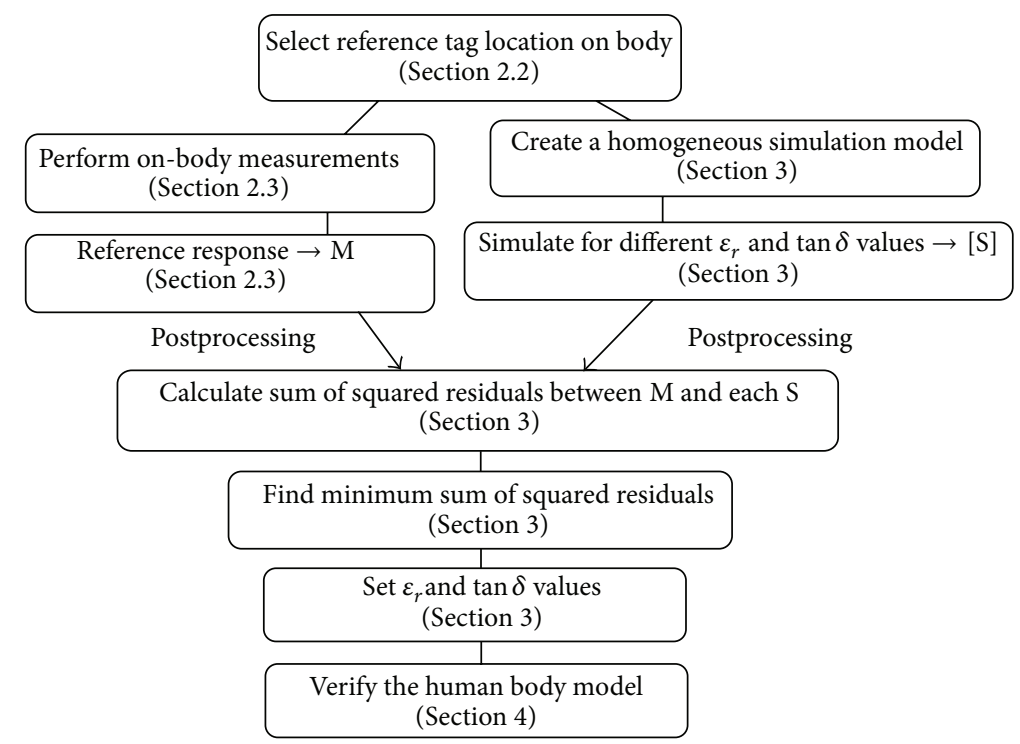

FIGURE 1: Flow chart for modeling the human body for RFID body-centric wireless systems. The human body model is developed using on-body measurements of a reference tag and electromagnetic simulations. The human body model electrical parameters $\varepsilon_{r}$ and tan $\delta$ are determined by calculating the sum of squared residuals between the measured reference response $\mathrm{M}$ and the simulated responses [S] attained for different $\varepsilon_{r}$ and $\tan \delta$ values.

create a homogeneous simulation model using a full-wave electromagnetic solver, ANSYS HFSS v15 [15], and define this model with the relative permittivity $\varepsilon_{r}$ and loss tangent $\tan \delta$. Finally, we set the values so that the sum of squared residuals between the measured and simulated reference responses is minimized. A flow chart of the proposed method is given in Figure 1.

The human body model is dependent on the tag location and the volunteer subject. However, the time to conduct a complete set of on-body measurements for a given tag location and human volunteer subject is rather short. This enables conducting fast measurements for different tag locations on body and for several human volunteer subjects, which allows one to create an average statistical catalog of human body models for each scenario. Creation of such catalog provides a practical, fast, and acceptably accurate engineering tool for initiating the design of optimized wearable antennas in bodycentric systems.

As summarized in Figure 1, this paper is structured as follows. In Section 2, we explain in detail the reference tag onbody measurement procedure. The setting of the parameters $\varepsilon_{r}$ and $\tan \delta$ is considered in Section 3. In Section 4 we demonstrate that the human body model can be utilized to estimate the performance of electrotextile wearable UHF RFID tag antennas. Section 5 concludes this paper with an outlook for future work.

\section{Reference Tag On-Body Measurements}

2.1. Reference Tag Antenna Design. Using ANSYS HFSS, we design a copper reference tag antenna in free-space operating in the UHF RFID frequencies and resonating at $950 \mathrm{MHz}$. The antenna is implemented on 0.126 mm-thick Kapton HN

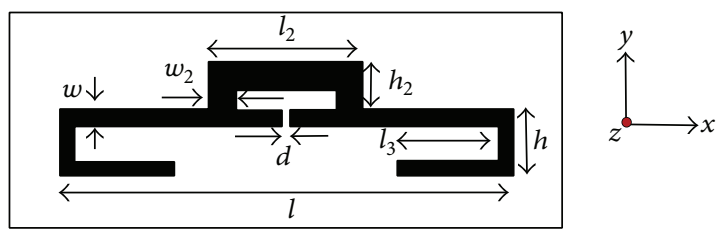

FIgURE 2: Dipole copper tag on Kapton substrate used as a reference tag for on-body measurements. The strap with the NXP IC [32] is attached over the $2 \mathrm{~mm}$ gap $d$ using conductive silver epoxy resin. The tag dimensions in $\mathrm{mm}$ are $l=90, l_{2}=30, l_{3}=20, w=3$, $w_{2}=5, h=13$, and $h_{2}=9$.

polyimide film. Its electrical properties $\left(\varepsilon_{r}=3.5\right.$ and $\tan \delta=$ $0.0026)$ are accurately specified, which makes it a suitable antenna for extracting the human model parameters. As seen from Figure 2, the reference antenna is a folded dipole equipped with a T-matching loop (parameters $l_{2}, w_{2}$, and $h_{2}$ ). Dipole antennas are simple single-layered structures with well-known impedance matching and miniaturization techniques. In addition, they enable easy integration with garments, which is necessary for their widespread use to become reality. We hence consider the dipole antenna as a proper choice for this study.

The loop transforms the tag antenna input impedance to inductive so that complex conjugate impedance matching between the antenna and the capacitive tag IC is achieved. The lengths $l$ and $l_{3}$ set the resonance frequency. The tag NXP IC has an equivalent input parallel resistance and capacitance of $2.85 \Omega$ and $0.9 \mathrm{pF}$, respectively, [16]. The overall goal in the free-space optimization is to achieve a high antenna realized gain $G_{\text {real }}$ at $950 \mathrm{MHz}$. This assures a valid response from the tag when operating on the human body even though severe 


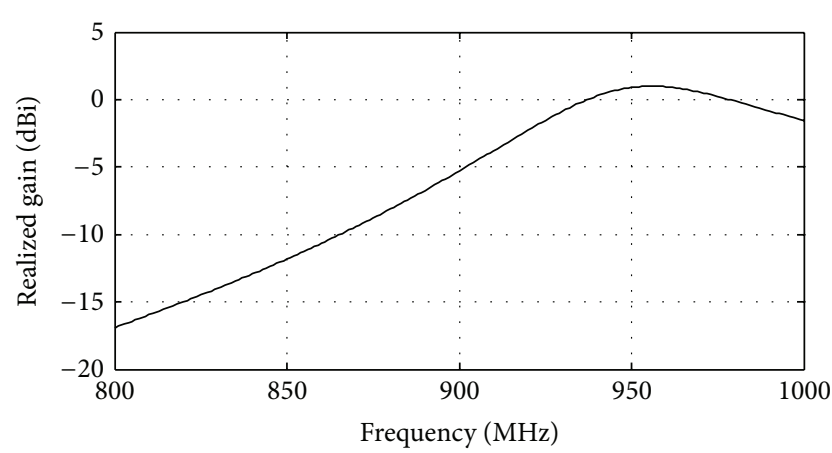

FIGURE 3: Reference tag realized gain in $+z$-axis in free-space.

performance degradation is encountered. The realized gain takes into account the antenna-IC impedance matching and is given by

$$
G_{\text {real }}(\theta, \phi)=\tau G(\theta, \phi),
$$

where $G(\theta, \phi)$ is the antenna gain and $\tau$ is the power transfer coefficient at the antenna $\left(P_{a}\right)$-IC $\left(P_{\text {ic }}\right)$ interface [17]:

$$
\tau=\frac{P_{\mathrm{ic}}}{P_{a}}=\frac{4 R_{a} R_{\mathrm{ic}}}{\left|Z_{a}+Z_{\mathrm{ic}}\right|},
$$

where $R_{a}$ and $R_{\mathrm{ic}}$ are the antenna and IC resistances, respectively, and $Z_{a}$ and $Z_{\mathrm{ic}}$ are the antenna and IC impedances, respectively. Equation (1) is evaluated by simulating the tag antenna properties and utilizing the NXP IC equivalent circuit model [16]. As seen from Figure 3, the desired high antenna realized gain at $950 \mathrm{MHz}$ is attained.

2.2. Case Studies: Upper Arm, Chest, and Head. Next, we conduct the reference tag on-body measurements. The asymmetric structure of the human body calls for careful selection of tag position in order to minimize shadowing effects and excessive power dissipation in the human tissues $[12,18]$. It is extremely difficult to assure that a single antenna is enough to realize an omnidirectional off-body link because at the UHF band the electromagnetic wave scattered from the tag antenna cannot propagate through the high-loss human body $[18,19]$. Here, we measured the reference tag response in the boresight direction without using any additional layers between the tag antenna and the human body. A UHF human body model was evaluated for three different locations on the human body: upper arm, chest, and head (Figure 4).

The upper arm and chest locations are suitable for applications where the antenna is intended to operate on the shirt or jacket. When the wearable antenna is integrated with a hat or hairband, a head model is needed for the antenna design and optimization processes. We conducted the reference measurements for two volunteer subjects to determine the variations in the human body model parameters $\varepsilon_{r}$ and $\tan \delta$ when the body proportions are changed. Most importantly, this provides valuable information about the acceptable accuracies related to the human body models.

2.3. Measurement Set-Up and Reference Response Results. We conducted the on-body measurements in an anechoic chamber environment. All measurements were performed in far-field conditions. The Voyantic RFID measurement system consists of a reader antenna, software, and measurement device [20]. The measurement device has adjustable output power and transmission frequency, which allows monitoring the tag reply under test to ISO $18000-6 \mathrm{C}$ query command. Core operations of this device are performed with vector signal analyzer. First, we utilized the system hardware calibration tag to wirelessly measure the path loss $L_{\text {iso }}$ in the wireless measurement channel. The path loss is defined from the transmitter's output port to the input port of a hypothetical polarization-matched isotropic antenna placed at the measurement plane. The channel characterization for one of the case studies is shown in Figure 5.

For each case we performed the channel characterization, after which we aligned the on-body attached reference tag at the measurement plane and monitored the minimum reader output threshold power $P_{\text {th }}$ required to attain a valid response from the tag in a polarization-matched configuration. The measurement set-up remained the same during channel characterization and during the threshold power measurement. The threshold power measurement set-ups for all the cases are shown in Figure 6.

Using the measured quantities $L_{\text {iso }}$ and $P_{\text {th }}$ we derive an expression for the measured read range. According to Friis equation, the power $P_{r}$ received by the tag antenna at a distance $r$ from the reader antenna is

$$
P_{r}=P_{t} G_{t} G_{r}\left(\frac{\lambda}{4 \pi r}\right)^{2}
$$

where $\lambda$ is the transmitted wavelength, $P_{t}$ is the power delivered to the reader antenna, and $G_{t}$ and $G_{r}$ are the gains of the reader and tag antenna, respectively. The received tag antenna power $P_{r}$ is only delivered to the tag IC when the tag antenna input impedance is conjugate-matched to the capacitive input of the tag IC. The tag IC minimum threshold power (sensitivity) $P_{\text {ic,th }}$ for activation is given by [21]

$$
P_{\text {ic }, \text { th }}=G_{\text {real }} L_{\text {iso }} P_{\text {th }} .
$$

Using (3) and (4) the forward link read range is attained as

$$
r_{\max }=\frac{\lambda}{4 \pi} \sqrt{\frac{P_{t} G_{t} G_{r} \tau}{P_{\text {ic,th }}}},
$$

where $P_{t} G_{t}=$ EIRP is the regulated isotropically radiated power ( $3.28 \mathrm{~W}$ in Europe). Solving for $G_{\text {real }}$ in (4) and substituting into (5) we arrive at the expression for the measured read range as follows:

$$
r_{\max }^{m}\left(P_{\mathrm{th}}\right)=\frac{\lambda}{4 \pi} \sqrt{\frac{\mathrm{EIRP}}{L_{\mathrm{iso}} P_{\mathrm{th}}}} .
$$

The threshold power at the measurement plane, that is, on tag $P_{\text {on-tag,th }}$, can be written as

$$
P_{\text {on-tag,th }}=L_{\text {iso }} P_{\text {th }} \text {. }
$$




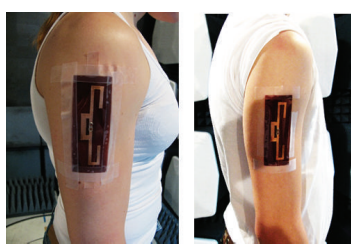

(a)

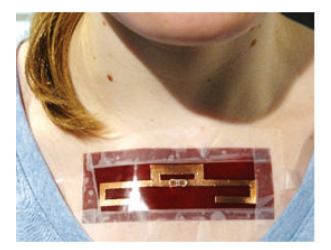

(b)

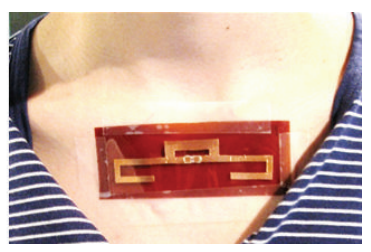

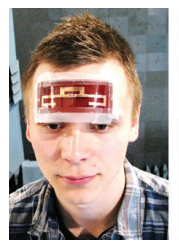

(c)

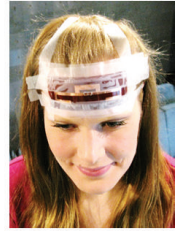

Figure 4: A human body model is evaluated for three different body locations: (a) upper arm, (b) chest, and (c) head. The tag antenna is linearly polarized. The tag is attached to the skin using adhesive tape.

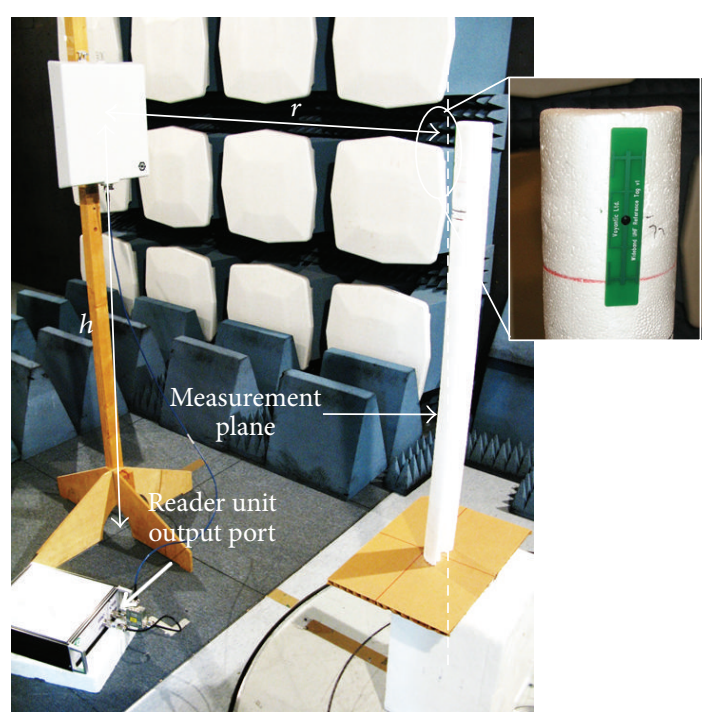

FIGURE 5: Channel characterization in anechoic chamber environment for the upper arm case. The calibration tag is polarizationmatched with the linearly (vertical) polarized reader antenna and located at the measurement plane in the far-field.

The measured path loss $L_{\text {iso }}$ follows from (7) as

$$
L_{\text {iso }}=\frac{\Lambda}{P_{\text {th }}^{*}},
$$

where $\Lambda$ is a known constant describing the sensitivity of the calibration tag and $P_{\mathrm{th}}^{*}$ is the measured threshold power of the calibration tag (the star refers to the calibration tag) in polarization-matched configuration. The measured quantities in (6) are thus $P_{\mathrm{th}}$ and $P_{\mathrm{th}}^{*}$.

For each of the cases, we repeated the $P_{\text {th }}$ measurement 20 times to determine the dynamic uncertainty. Between the measurement repetitions, the volunteer subject wearing the reference tag was moved from the measurement position and then repositioned for the boresight measurement.

From the 20 repeated threshold measurements, we computed the sample average $(u)$ and sample standard deviation $(v)$ of $P_{\mathrm{th}}$ to estimate the expectation and standard deviation of the governing probability distribution. To estimate how the measurement uncertainty propagates from the measured threshold power to the theoretical read range through the nonlinear equation (6), we linearized it in the neighborhood of the measured sample average using the first order Taylor
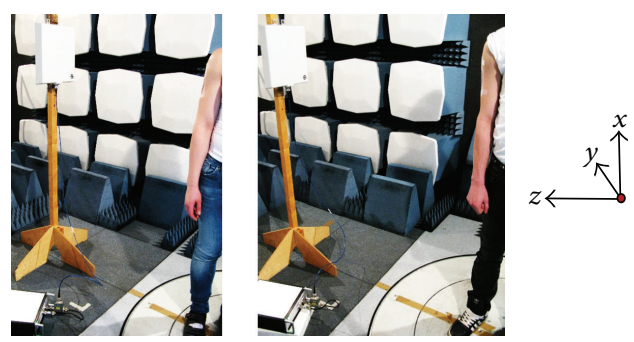

(a)
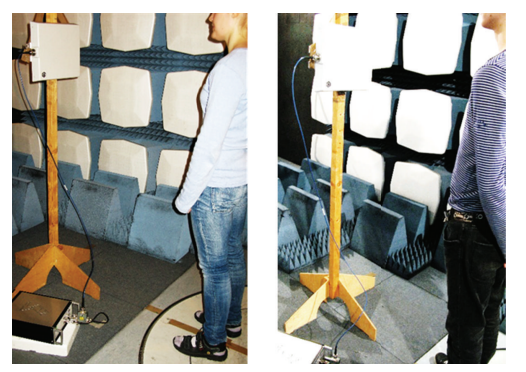

$z \longleftarrow q_{x}$

(b)
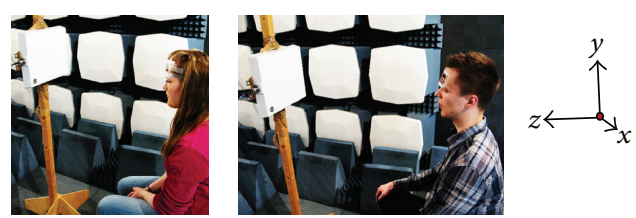

(c)

FIGURE 6: Reference tag boresight threshold power $P_{\text {th }}$ measurement for the studied cases: (a) upper arm case, $H$-plane ( $z y$-plane) measurement; (b) chest, $E$-plane ( $z x$-plane) measurement; and (c) head, $E$-plane ( $z x$-plane) measurement.

expansion. As a result, we obtained the first order approximations

$$
r_{\max }^{m}(u), \quad r_{\max }^{m}(u) \frac{v}{2 u}=\sigma,
$$

of the expectation and standard deviation of $r_{\max }^{m}$, respectively. The average read ranges (Nom) based on the $P_{\text {th }}$ measurements and the standard deviations $( \pm \sigma)$ are shown in Figures 7, 8, and 9.

For all the measurement cases the standard deviation is low, implying that the dynamic uncertainty is low. The chest location provides a relatively flat platform for the tag antenna and the chest local tissue properties do not differ significantly 


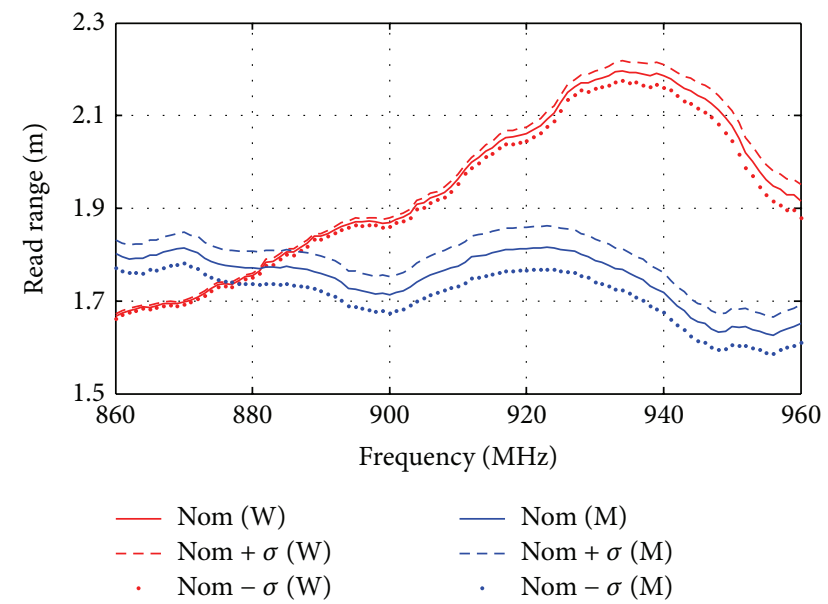

FIGURE 7: Reference response measurement results ( $H$-plane) for the upper arm case for woman (W) and man (M). The measurements are based on 20 repetitive threshold power measurements.

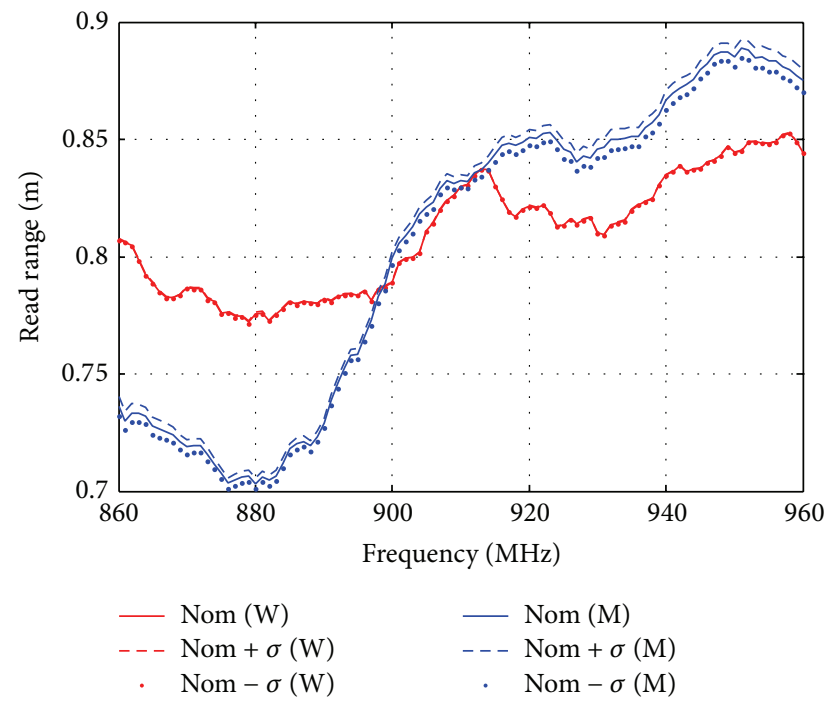

FIGURE 8: Reference response measurement results ( $E$-plane) for the chest case for woman (W) and man (M). The measurements are based on 20 repetitive threshold power measurements.

between the volunteer subjects. We hence observe good agreement between the reference responses for the volunteer subjects. Both the arm and head provide round platforms. Relatively large differences are expected in the local arm and head tissue properties between the volunteer subjects. Further, the radius of the arm and head curvatures are different for the volunteer subjects. We see in Figures 7 and 9 that this results in somewhat different reference responses between the subjects.

In summary, the reference tag response is dependent on the volunteer subject and the tag antenna location on the human body. According to the measurement results, we expect a difference of maximum 0.5 meters between the measured reference responses from the two test subjects based on 20 repeated measurements.

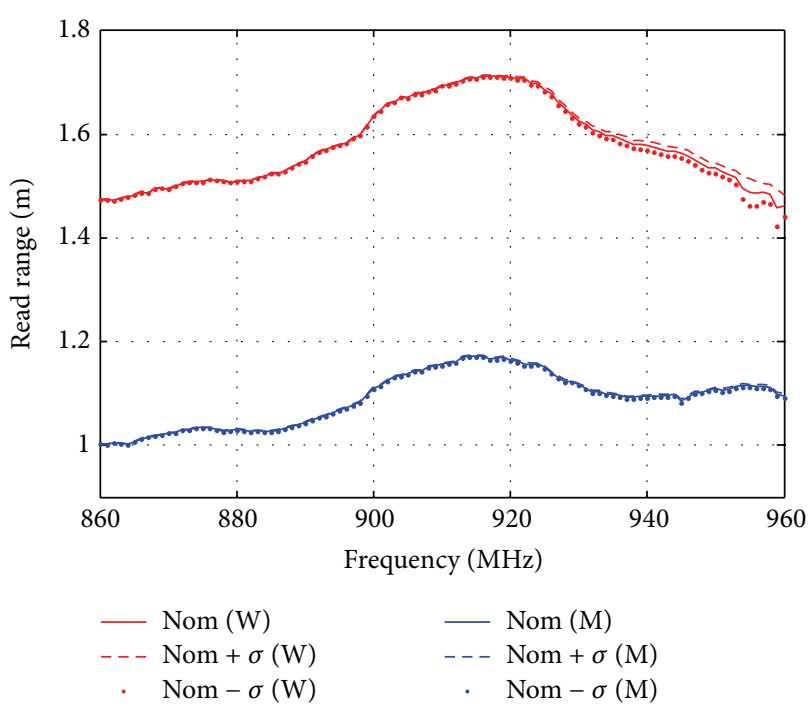

FIGURE 9: Reference response measurement results (E-plane) for the head case for woman (W) and man (M). The measurements are based on 20 repetitive threshold power measurements.

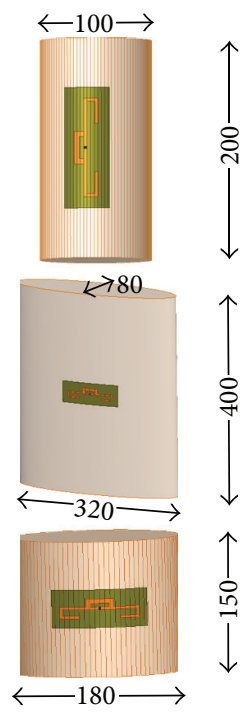

FIGURE 10: Homogeneous simulation models for (up to down): the upper arm, chest, and head cases. Dimensions are in $\mathrm{mm}$. The same model dimensions are used for both volunteer subjects.

\section{Reference Tag On-Body Simulations}

For each case, we created a homogeneous simulation model that represents the human body part where the reference tag is located (Figure 10). We used the same model dimensions for both volunteer subjects. This way one fixed human body model represents the tag location, and the model parameters $\varepsilon_{r}$ and $\tan \delta$ will count for the differences in body proportions and properties between different individuals. A wearable antenna designer would hence in future select the model dimensions according to the body-centric application 


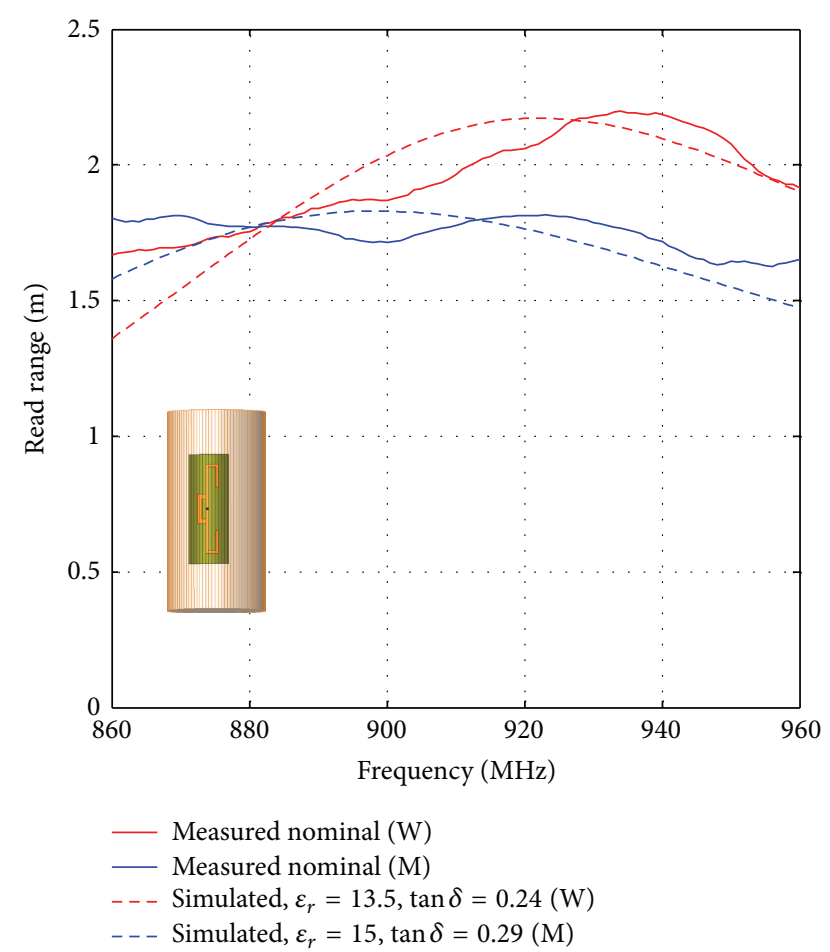

FIGURE 11: Measured and simulated reference tag on human arm and human arm model, respectively.

(tag location) and then set the model parameters $\varepsilon_{r}$ and $\tan \delta$ according to the target group (volunteer subjects).

We used cylindrical models for the upper arm and head cases. For the chest we chose an elliptical model. We assigned realistic dimensions for each model to mimic the body part. In a polarization-matched configuration the simulated read range is calculated from (5).

The tag performance will be mostly influenced by the outer tissue layers. For each configuration in Figure 10, we swept the relative permittivity from 5 to 50 with 0.5 step and the loss tangent from 0.2 to 0.5 with 0.01 step to cover the permittivities and loss tangents measured from human tissues [22]. Referring to Figures 7-9, we expect that at least for the arm and head cases, the human body model $\varepsilon_{r}$ and $\tan \delta$ values will differ between the two volunteer subjects.

For each studied case and volunteer subject, we set the $\varepsilon_{r}$ and $\tan \delta$ values so that the sum of squared residuals is minimized between the averaged measured and the simulated read ranges. The average measured read range $g\left(f_{i}, d_{i}\right)$ consists of $n$ data pairs, where $i=1, \ldots, n$. Here $n$ equals the number of frequency points (101) used in the simulations $\left(860-960 \mathrm{MHz}, 1 \mathrm{MHz}\right.$ step). The pair $\left(f_{i}, d_{i}\right)$ consists of the frequency variable $f_{i}$ and the average measured read range variable $d_{i}$. Each of the simulated read range solutions $f\left(f_{i}, \beta\right)$ holds a vector $\beta$ with the unique parameter combination of $\varepsilon_{r}$ and $\tan \delta$. The sum of squared residuals is calculated as

$$
S=\sum_{i=1}^{n} r_{i}^{2}
$$

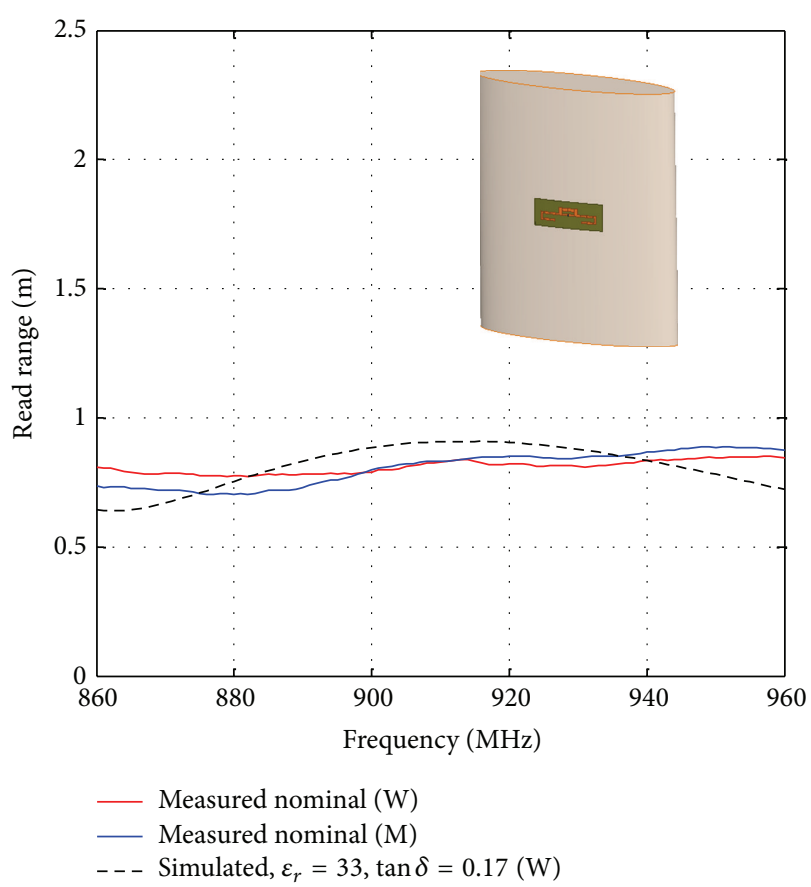

FIGURE 12: Measured and simulated reference tag on human chest and human chest model, respectively.

where the residual $r_{i}$ is defined as the difference between the measured and simulated read range values such that

$$
r_{i}=d_{i}-f\left(f_{i}, \beta\right) .
$$

We observe from Figures 11, 12, and 13 that the simulated read ranges are well within the accepted ranges, that is, \pm 0.5 meter from the average measured read ranges.

We observe that for a given case the model parameters $\varepsilon_{r}$ and $\tan \delta$ may differ significantly between different volunteer subjects. This supports our statement that application and case specific human body models for body-centric RFID are needed. A general body model will not provide an optimum tool for wearable antenna design.

The skin is the first and most prominent human tissue affecting the tag performance for all studied cases. Still, the measurement results in Figures 11-13 show that the reference tag performance encounters the largest degradation when located on the chest. We remember that the reference tag response is not only affected by the human tissues, but also by the air between the tag and body and the structure of the body surface. It is obvious from the measurement set-up in Figure 4 that the round arm and head locations will add air between the tag and the body, which decreases the human body effects on the tag performance.

It should be noted that the attained $\varepsilon_{r}$ and $\tan \delta$ values are not only dependent on the measured reference response, but also on the chosen human body model. Therefore, the same shape for the human body model should be used for future antenna design and optimization and the antenna on-body measurements should be conducted in similar manner as for the reference tag. The total time to complete one set of measurements for a given human subject and antenna location to 


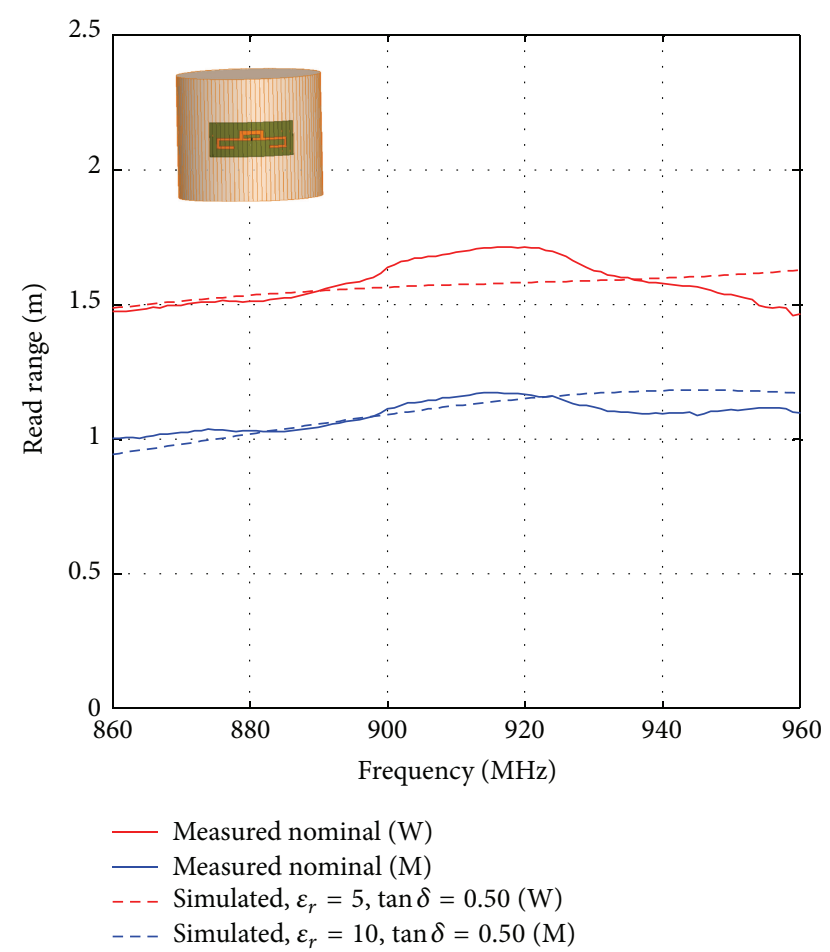

Figure 13: Measured and simulated reference tag on human head and human head model, respectively.

attain the human body model parameters is rather short. This allows creating a catalog of parameters for different scenarios and body models. Such catalog would augment the future development of new and efficient wearable antenna solutions in body-centric communication applications.

\section{Human Body Model Verification}

4.1. Electrotextile Antennas. We verify the human body models by measuring and simulating the read ranges of wearable tags on human body and on the human body models, respectively.

In recent studies, electrotextiles are demonstrated to be competitive solutions for the realization of wearable antennas [9, 23-27]. Electrotextile technologies are flexible conductive materials that are constructed by interpolating metal or polymer threads with conventional fabric thread or conductive threads [25]. Commercially available conductive fabrics and embroidered structures are commonly used as electrotextiles in wireless body-centric applications. These are believed to be a strong candidate for body-worn electronics thanks to their washability, durability, flexibility, and seamless integrability [25].

Embroidered structures are challenging materials to model. Compared to solid conventional antenna materials, such as bulk copper, embroidered structures are anisotropic materials. Their electrical properties are strongly dependent on the stitching density and pattern [26]. Further, the thickness of the embroidered structure is not unambiguously defined, as it depends on the stitching density and as the
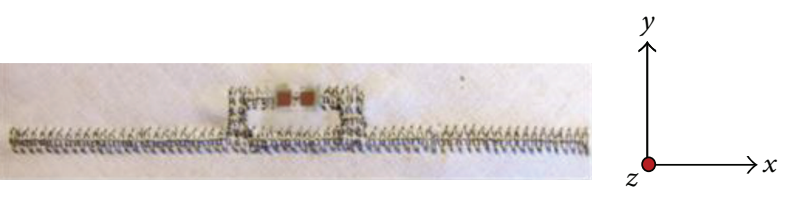

(a)
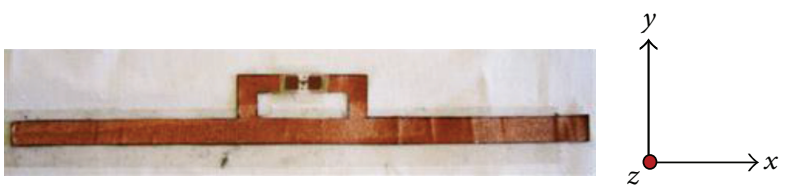

(b)

FIGURE 14: Electrotextile tags used for the human body model verification: (a) embroidered tag antenna and (b) fabric tag antenna. A NXP IC is attached using conductive silver epoxy resin.

embroidery machine creates the structure on both sides of the flexible fabric substrate [27].

Our electrotextile RFID tag antennas used for the human body model verification are presented in Figure 14. Both antennas were implemented on cotton fabric $\left(\varepsilon_{r}=1.8\right.$ and $\tan \delta=0.018)$. The embroidered antenna was fabricated [27] using conductive thread [28]. The thread is created from two strands of conductive fibers, which are each in turn created from 34 filaments. The filaments have a polyamide core plated with silver. The thread dc resistance is $500 \Omega / \mathrm{m} \pm 100 \Omega / \mathrm{m}$. The other tag antenna uses commercially available [29] conductive copper polyester taffeta fabric (35\% copper).

Previously, we demonstrated that the embroidered and fabric antennas can be modeled as infinitely thin homogeneous conductors with a sheet resistance value of $1.25 \Omega / \mathrm{sq}$. and $0.40 \Omega$ /sq., respectively [30]. This technique eliminates the uncertainty related to the electrotextile thickness, as the sheet resistance is independent of the thickness.

4.2. Case Studies: Upper Arm, Chest, and Head. We simulated the electrotextile antennas on the human body models attained in Section 3 and conducted the on-body measurements similarly as presented in Section 2.3. The measured and simulated boresight ( $+z$-direction) read ranges for all the cases are presented in Figures 15-20.

4.3. Discussion. We clearly see that the wearable tag performs better on the female body: we may expect up to 1 meter more read range on the female body. The head and the chest are high-loss platforms for the antenna. This, together with the high-loss sewing thread creating the embroidered tag, lowers the embroidered tag antenna quality factor. A low quality factor results in a large bandwidth, which can be seen from Figures 18 and 20. Also the fabric tag shows a relatively large bandwidth on these high-loss platforms (Figures 17 and 19). Due to the low sheet resistance value of the copper fabric we observe for all the cases a slightly better read range for the fabric tag compared to the embroidered tag. 


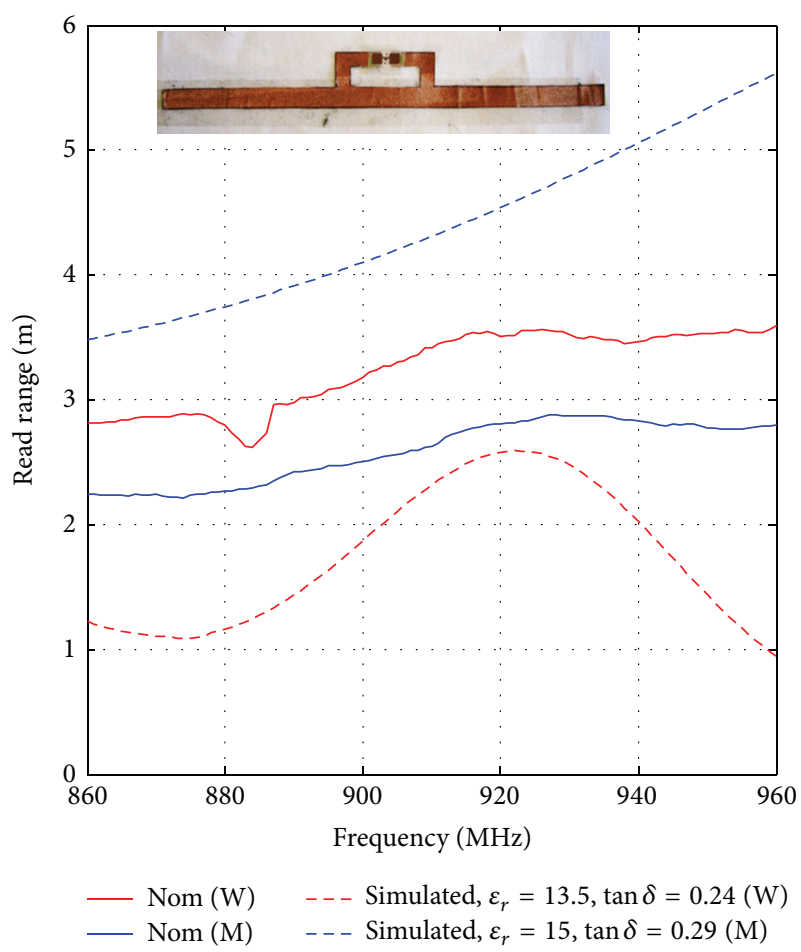

FIGURE 15: Measured and simulated fabric tag on human arm and human arm model, respectively.

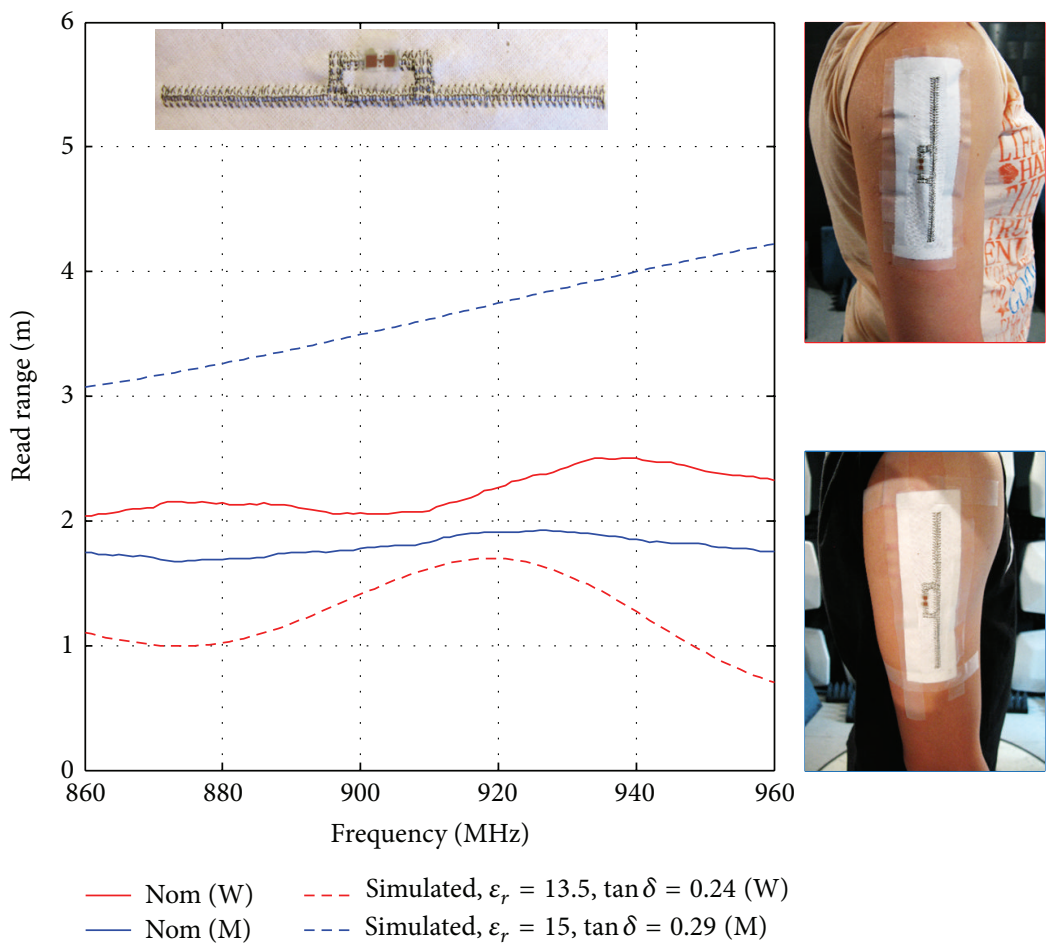

FIGURE 16: Measured and simulated embroidered tag on human arm and human arm model, respectively. 


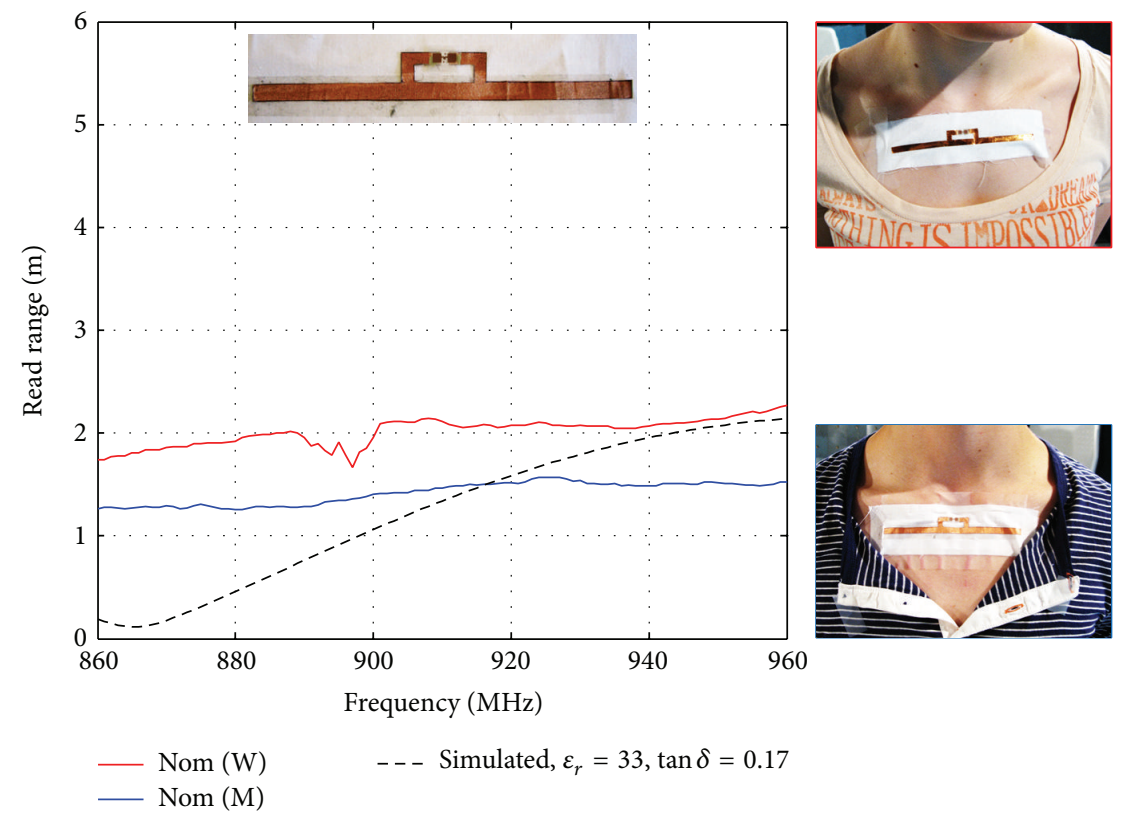

FIGURE 17: Measured and simulated fabric tag on human chest and on human chest model, respectively.

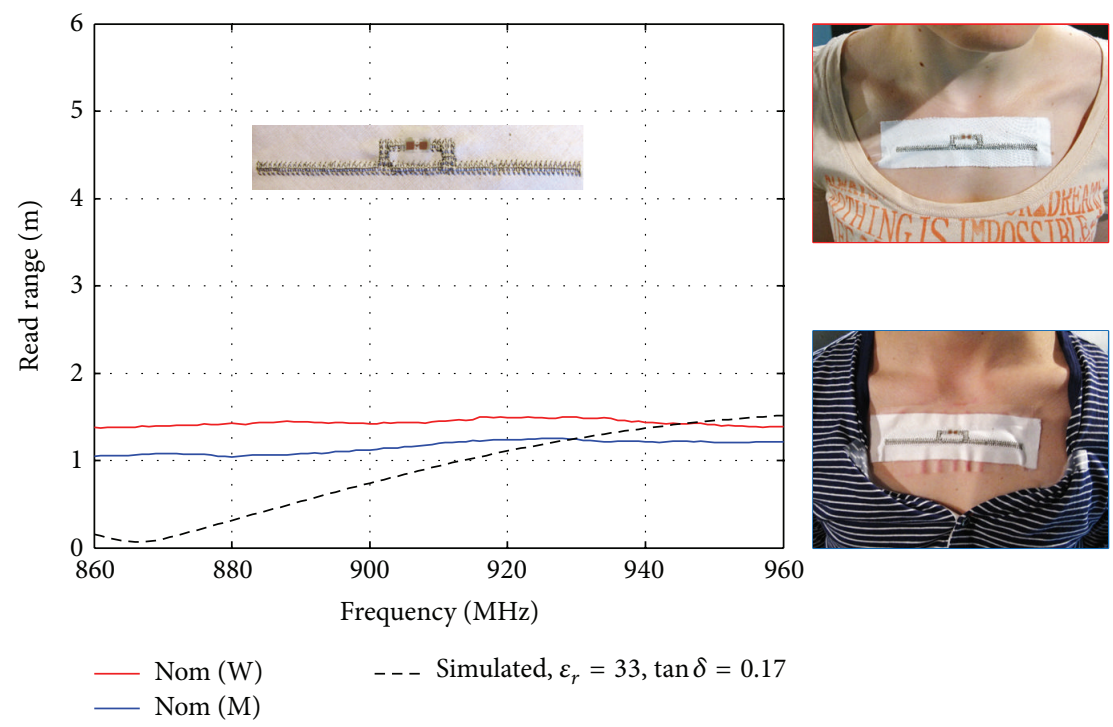

Figure 18: Measured and simulated embroidered tag on human chest and on human chest model, respectively.

Before evaluating the agreement between simulation and measurements results, we should consider four major uncertainty sources.

(1) The human body model $\varepsilon_{r}$ and $\tan \delta$ parameters are strongly correlated with tag location on body, volunteer subject, and the tag physical orientation and attachment on the body. It is extremely difficult to attach the electrotextile tags at identical locations and orientations on body as the reference tag.

(2) Compared to the reference tag antenna materials, the embroidered electrotextiles are challenging to model.
In [30] we demonstrated that electrotextile dipoletype tag antennas can be modeled with \pm 1.5 meter read range accuracy.

(3) The electrotextile antennas have limited fabrication accuracy. According to our knowledge and previous work [30, 31], the electrotextile antenna fabrication process can result in \pm 1 meter free-space read range difference between two antenna samples.

(4) The parameters $L_{\text {iso }}$ and $P_{\text {th }}$ have limited measurement accuracies. We are able to measure $P_{\text {th }}$ with $\pm 0.2 \mathrm{~dB}$ accuracy and $L_{\text {iso }}$ with $-0.45 \cdots-0.05 \mathrm{~dB}$ accuracy [20]. 

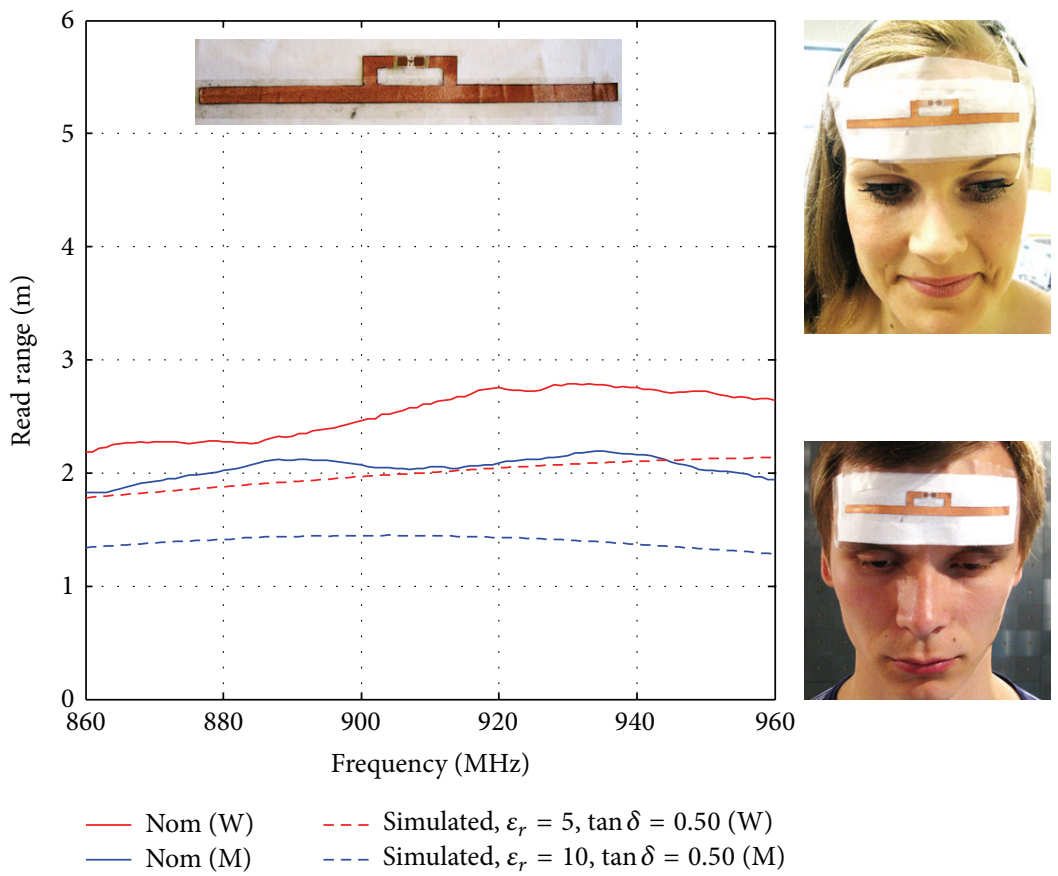

FIgURE 19: Measured and simulated fabric tag on human head and on human head model, respectively.

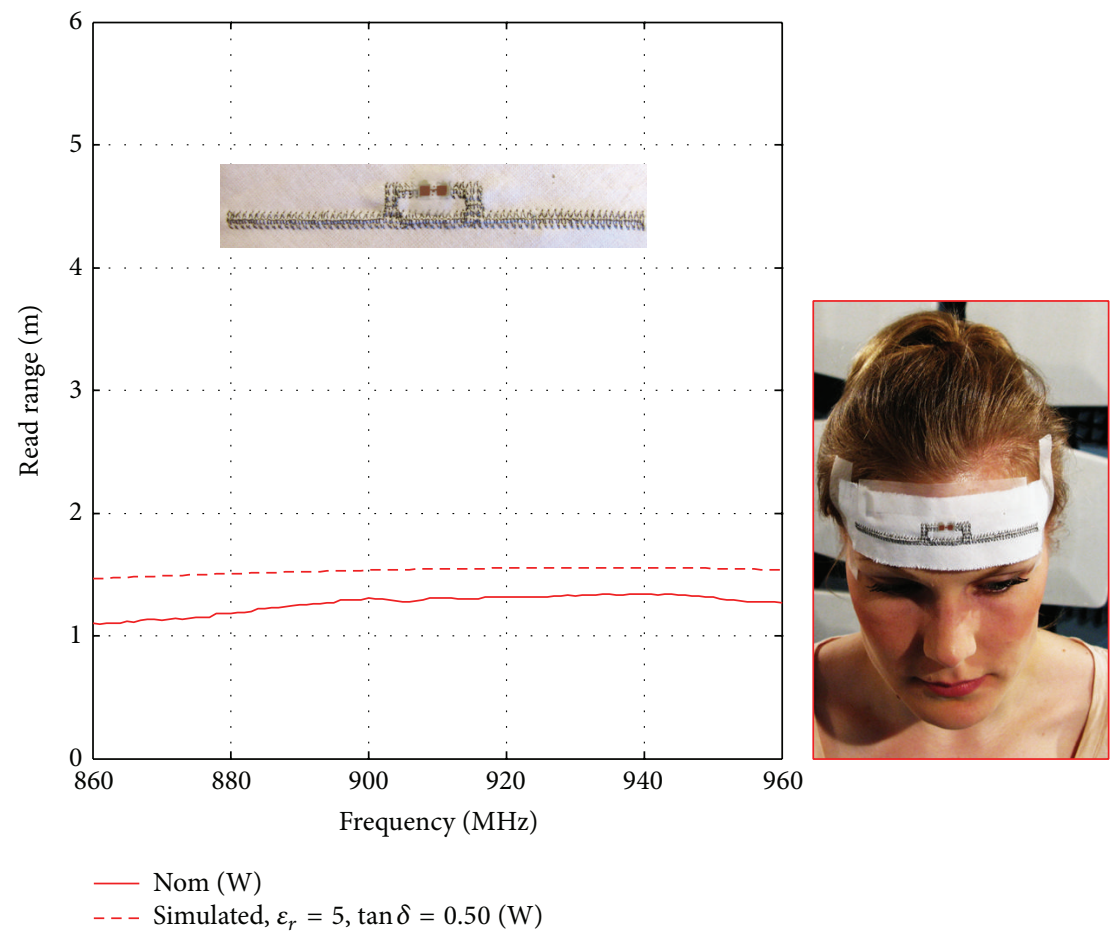

FIGURE 20: Measured and simulated embroidered tag on human head and on human head model, respectively. The embroidered tag is not responding on the male human head.

The impact of the first uncertainty source is dependent on the tag location on body, while the other three uncertainty sources are independent of the measurement set-up. When considering Figures 15 and 16, we notice a maximum difference of almost 3 meters between measured and simulated responses. In measurement point of view, the human arm is a challenging antenna platform. In this case, the first listed uncertainty source is strongly influencing the results. The curvature of the human arm makes it difficult to align the flexible electrotextile tag antenna in the same way as 
the reference tag antenna. Unfortunately, this practical issue is typically present when the human body constitutes the antenna platform and the antenna location is on curved surface. One way to overcome some of the issues is to use simplified phantoms for measurement purpose. However, this approach would not exclude the practical issues when the wearable antenna is put into operation in its intended bodycentric application.

For the chest and head cases (Figures 17-20) we attain better agreement between measured and simulated results. In these cases we were able to align the electrotextile tag antennas in a similar way to the reference tag thanks to the less curved platforms.

When evaluating a human body model for body-centric applications we should always take the uncertainties into consideration. Hence, the tag performance prediction we attain using our human body models should not be considered as an absolute truth, but instead as a guideline for initiating the design of optimized wearable antennas in body-centric systems. As our modeling approach can be quickly and easily adopted in practice, it will assist the future development of new and efficient wearable antenna solutions.

\section{Conclusion and Future Work}

In this paper, we proposed a method for modeling the human body in RFID body-centric wireless systems. The method eliminates the need for knowing the electrical properties of specific biological tissues and it can be effectively adopted in practice for different body-centric scenarios. The paper also considered practical aspects and issues of on-body measurements and provided important information about how different human bodies will influence the wearable tag antenna performance. The paper discussed practical limitations of the proposed modeling method and elaborated on the accepted human body model accuracies. The human model will be utilized in future electrotextile antenna design and optimization.

\section{Conflict of Interests}

The authors declare that there is no conflict of interests regarding the publication of this paper.

\section{Acknowledgments}

The authors wish to thank Dr. A. Ali Babar and Mikko Toivonen for their valuable support in conducting the measurements.

\section{References}

[1] C. Occhiuzzi and G. Marrocco, "The RFID technology for neurosciences: feasibility of Limbs' monitoring in sleep diseases," IEEE Transactions on Information Technology in Biomedicine, vol. 14, no. 1, pp. 37-43, 2010.

[2] H. Rajagopalan and Y. Rahmat-Samii, "Ingestible RFID biocapsule tag design for medical monitoring," in Proceedings of the IEEE International Symposium on Antennas and Propagation and CNC-USNC/URSI Radio Science Meeting (AP-S/URSI '10), Toronto, ON, Canada, July 2010.

[3] Y. Li, R. Vyas, A. Rida, J. Pan, and M. M. Tentzeris, "Wearable RFID-enabled sensor nodes for biomedical applications," in Proceedings of the 58th Electronic Components and Technology Conference (ECTC '08), pp. 2156-2159, Lake Buena Vista, Fla, USA, May 2008.

[4] C. Occhiuzzi, S. Cippitelli, and G. Marrocco, "Modeling, design and experimentation of wearable RFID sensor tag," IEEE Transactions on Antennas and Propagation, vol. 58, no. 8, pp. 2490-2498, 2010.

[5] G. Marrocco, "RFID antennas for the UHF remote monitoring of human subjects," IEEE Transactions on Antennas and Propagation, vol. 55, no. 6, pp. 1862-1870, 2007.

[6] J. Kim and Y. Rahmat-Samii, "Implanted antennas inside a human body: simulations, designs, and characterizations," IEEE Transactions on Microwave Theory and Techniques, vol. 52, no. 8, pp. 1934-1943, 2004.

[7] Y. Rahmat-Samii and J. Kim, Implanted Antennas in Medical Wireless Communications, Synthesis Lectures on Antennas, Morgan \& Claypool, 2006.

[8] D. Psychoudakis, G.-Y. Lee, C.-C. Chen, and J. L. Volakis, "Military UHF body-worn antennas for armored vests," in Proceedings of the 4th European Conference on Antennas and Propagation (EuCAP '10), pp. 1-4, Barcelona, Spain, April 2010.

[9] C. Hertleer, H. Rogier, L. Vallozzi, and L. van Langenhove, "A textile antenna for off-body communication integrated into protective clothing for firefighters," IEEE Transactions on Antennas and Propagation, vol. 57, no. 4, pp. 919-925, 2009.

[10] T. Kellomäki and L. Ukkonen, "Design approaches for bodyworn RFID tags," in Proceedings of the ISABEL Conference, pp. 7-10, Rome, Italy, 2010.

[11] H. Rajagopalan and Y. Rahmat-Samii, "Conformal RFID antenna design suitable for human monitoring and metallic platforms," in Proceedings of the 4th European Conference on Antennas and Propagation (EuCAP '10), pp. 1-5, Barcelona, Spain, April 2010.

[12] T. Kellomäki, T. Björninen, L. Ukkonen, and L. Sydänheimo, "Shirt collar tag for wearable UHF RFID systems," in Proceedings of the 4th European Conference on Antennas and Propagation (EuCAP '10), pp. 1-5, Barcelona, Spain, 2010.

[13] Nello Carrara, IFAC-CNR, Italy, "Dielectric Properties of Body Tissues," 2012, http://niremf.ifac.cnr.it/tissprop/ htmlclie/htmlclie.htm.

[14] M. A. Ziai and J. C. Batchelor, "Temporary on-skin passive UHF RFID transfer tag," IEEE Transactions on Antennas and Propagation, vol. 59, no. 10, pp. 3565-3571, 2011.

[15] ANSYS HFSS, 2013, http://www.ansys.com/.

[16] T. Björninen, L. Sydänheimo, and L. Ukkonen, "Development and validation of an equivalent circuit model for UHF RFIC IC based on wireless tag measurements," in Proceedings of the AMTA Symposium, p. 6, Bellevue, Wash, USA, 2012.

[17] K. V. S. Rao, P. V. Nikitin, and S. F. Lam, "Impedance matching concepts in RFID transponder design," in Proceedings of the 4th IEEE AutoID, pp. 39-42, Buffalo, NY, USA, October 2005.

[18] S. Manzari, C. Occhiuzzi, and G. Marrocco, "Reading range of wearable textile RFID tags in real configurations," in Proceedings of the 5th European Conference on Antennas and Propagation (EUCAP '11), pp. 433-436, Rome, Italy, April 2011.

[19] T. Kellomäki, J. Heikkinen, and M. Kivikoski, "One-layer GPS antennas perform well near a human body," in Proceedings of 
the European Conference on Antennas and Propagation (EUCAP '07), Edinburgh, UK, 2007.

[20] Voyantic, Ltd., Espoo, Finland, 2013, http://www.voyantic.com.

[21] T. Björninen, M. Lauri, K. Koski et al., "Wireless measurement of wake-up power and impedance of UHF RFID IC," in Proceedings of the 33rd AMTA Symposium, pp. 63-68, Englewood, Colo, USA, 2011.

[22] IFAC-CNR, Florence, Italy, "Dielectric Properties of Body Tissues," 2012, http://niremf.ifac.cnr.it/tissprop/htmlclie/ htmlclie.htm.

[23] J. S. Roh, Y. S. Chi, J. H. Lee, Y. Tak, S. Nam, and T. J. Kang, "Embroidered wearable multiresonant folded dipole antenna for FM reception," IEEE Antennas and Wireless Propagation Letters, vol. 9, pp. 803-806, 2010.

[24] Y. Bayram, Z. Yijun, S. S. Bong et al., "E-textile conductors and polymer composites for conformal lightweight antennas," IEEE Transactions on Antennas and Propagation, vol. 58, no. 8, pp. 2732-2736, 2010.

[25] Y. Ouyang and W. J. Chappell, "High frequency properties of electro-textiles for wearable antenna applications," IEEE Transactions on Antennas and Propagation, vol. 56, no. 2, pp. 381-389, 2008.

[26] E. Moradi, T. Björninen, L. Ukkonen, and Y. Rahmat-Samii, "Characterization of embroidered dipole-type RFID tag antennas," in Proceedings of the RFID-TA Conference, Nice, France, 2012.

[27] E. Koski, K. Koski, T. Björninen, A. A. Babar, L. Sydänheimo, and L. Ukkonen, "Fabrication of embroidered UHF RFID tags," in Proceedings of the IEEE AP-S Symposium, Chicago, Ill, USA, 2012.

[28] Shieldex 110f34 dtex 2-ply HC, "High grade multifilament Nylon yarn," 2013, http://www.statex.de.

[29] LessEMF, EMF Shielding \& Conductive Fabrics, 2013, http://www.lessemf.com/fabric.html.

[30] K. Koski, E. Moradi, A. Vena et al., "Characterization of electro-textiles using wireless reflectometry for optimization of wearable UHF RFID tags," in Proceedings of the 34th PIERS Symposium, Stockholm, Sweden, 2013.

[31] K. Koski, E. Moradi, and A. Ali Babar, "Durability of embroidered antennas in wireless body-centric health applications," in Proceedings of the European Conference on Antennas and Propagation (EuCAP '13), Gothenburg, Sweden, 2013.

[32] NXP Semiconductors, "Product Data Sheet," 2012, http://www.nxp.com/documents/data_sheet/SL3S1203_1213 .pdf. 

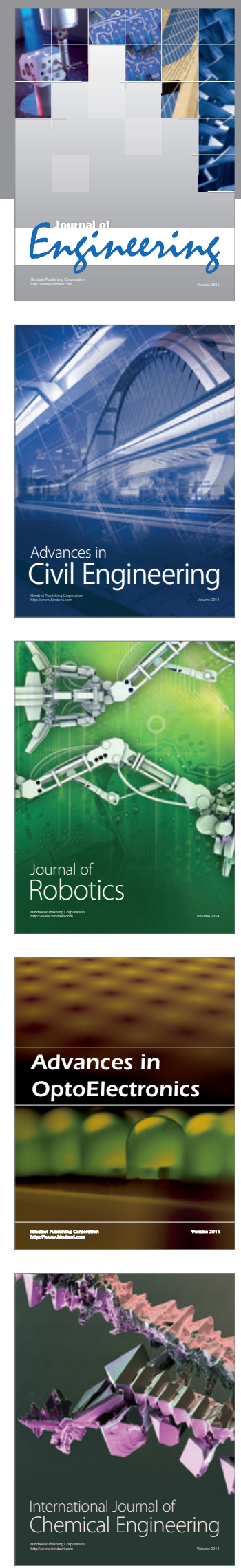

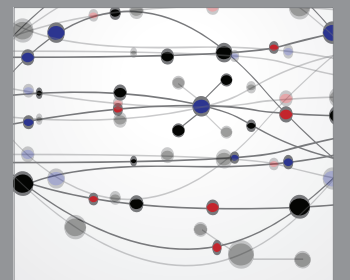

The Scientific World Journal
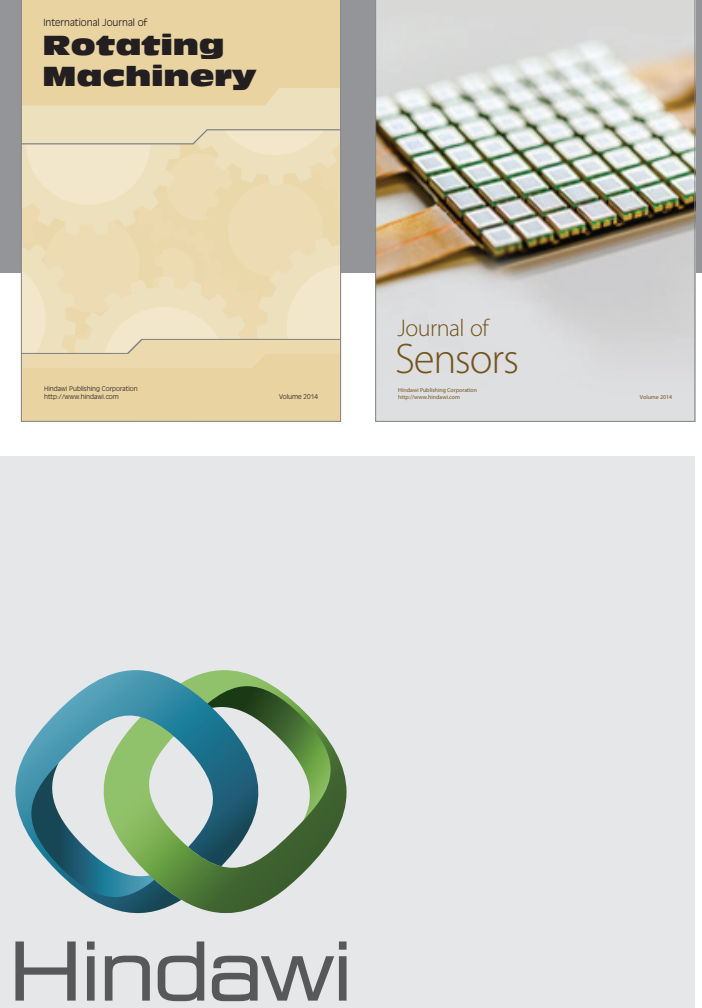

Submit your manuscripts at http://www.hindawi.com
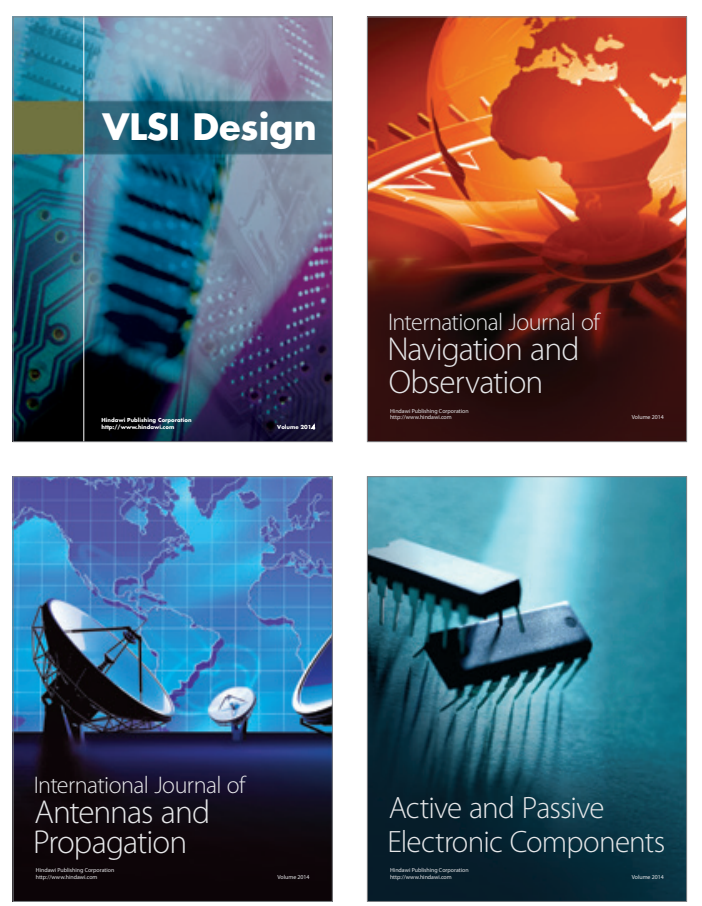
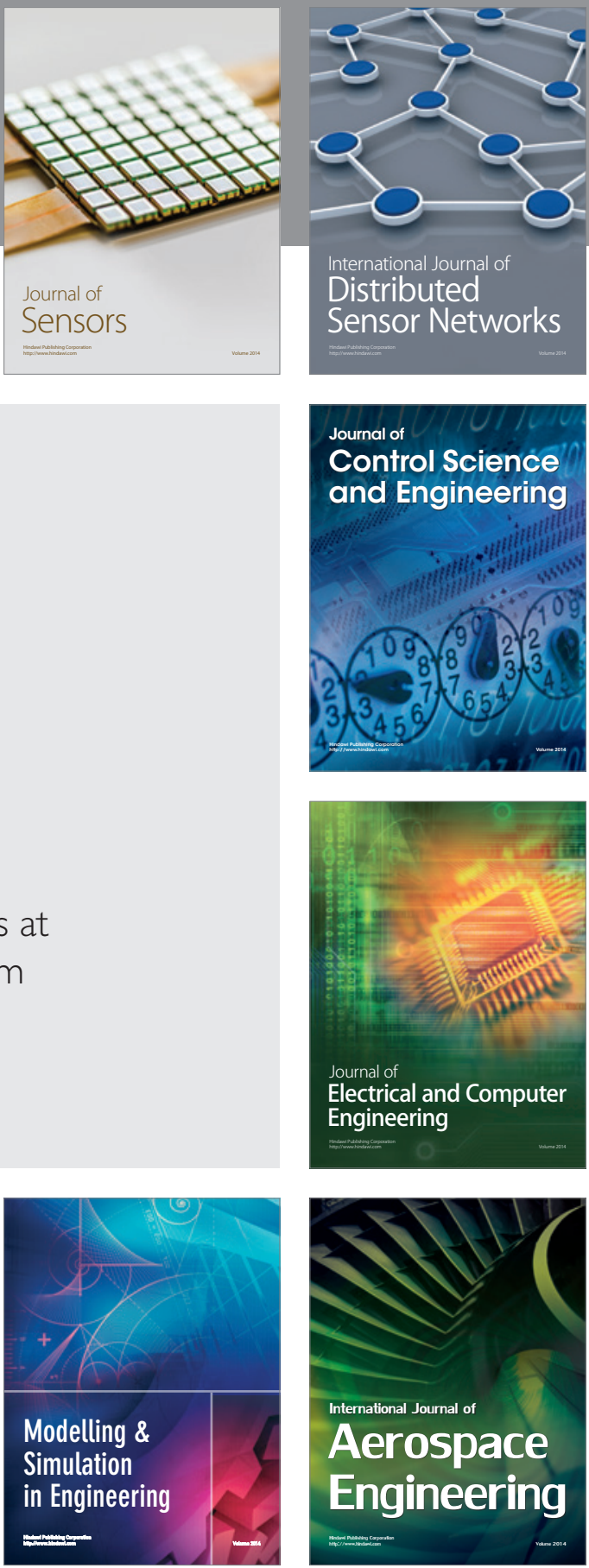

Journal of

Control Science

and Engineering
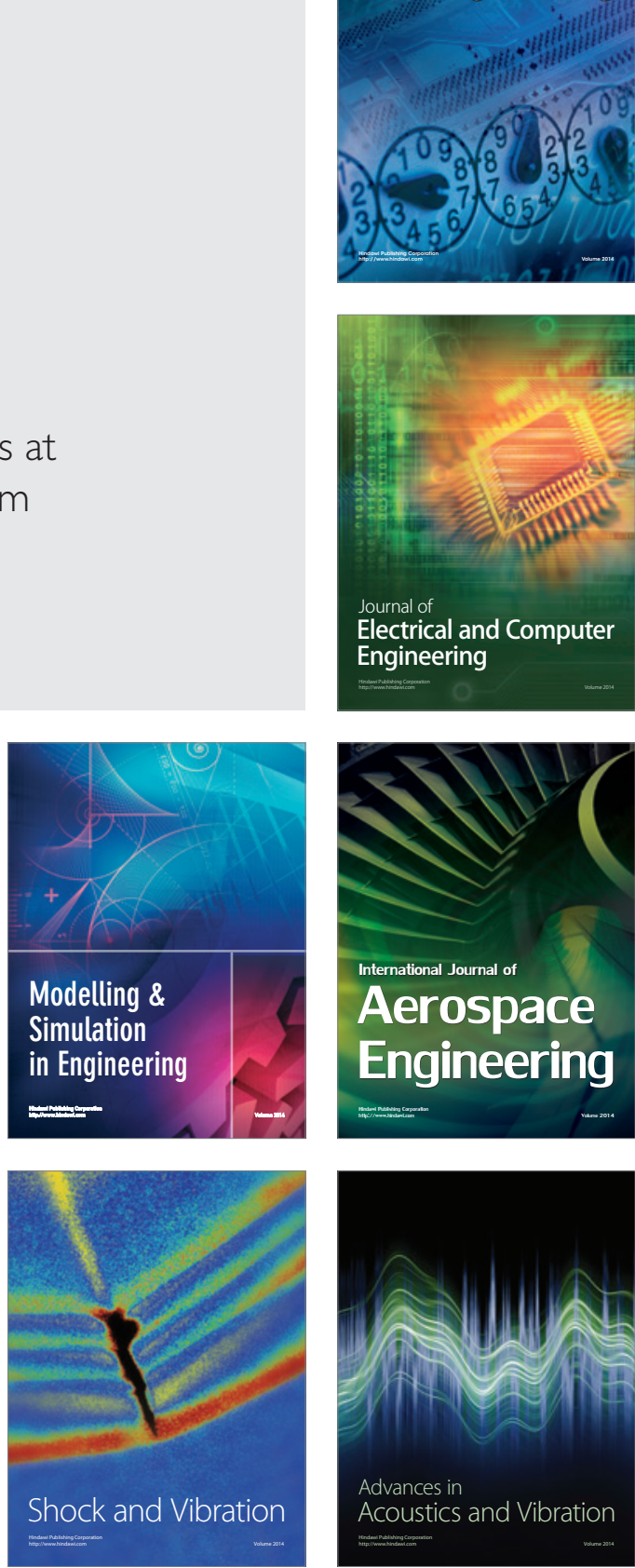